\title{
Gama Radyasyonunun, Kalp-Damar Tedavisinde Kullanılan Losartan Potasyum ve İndapamid İlaçlarında Oluşturduğu Paramanyetik Merkezin EPR Spektroskopisi ile İncelenmesi
}

\author{
Yunus Emre OSMANOĞLU \\ Dicle Üniversitesi, Tıp Fakültesi, Diyarbakır, Türkiye \\ Geliş / Received: 21/06/2018, Kabul / Accepted: 24/10/2018
}

\begin{abstract}
$\ddot{O} \mathbf{z}$
Bu çalıșmada, losartan potasyum ve indapamide ilaçlarında gama radyasyonu ile oluşturulan serbest radikallerin doğası elektron paramanyetik rezonans (EPR) spektroskopisi kullanılarak oda sıcaklığında belirlenmiştir. $\mathrm{Bu}$ örneklerin EPR spektrumları sırasıyla $\mathrm{CHCHCH}_{3}$ ve $\dot{\mathrm{C}} \mathrm{HCHCH}_{3} \mathrm{NH}$ radikallerine atfedilmiştir. Serbest radikallerin aşırı ince yapı sabitleri ve g-değerleri hesaplanmıştır. EPR ölçümleri radyasyondan sonra oluşan paramanyetik merkezin kararlılığı ve spin yoğunluluğunun absorplanan radyasyonun dozuna bağlı olduğunu göstermiştir. Her iki örnekte oluşan paramanyetik merkezler örneklerin kimyasal yapısı göz önüne alınarak belirlenmiştir. İki materyalin manyetik özellikleri ve paramanyetik merkezin mümkün yapısı bu çalışmada tartışılmış̧ır.
\end{abstract}

Anahtar Kelimeler: EPR, Gama radyasyonu, Paramanyetik Merkez

\section{Investigation of Paramagnetic Center Formed by Gamma Radiation in Losartan Potassium and Indapamide Drugs Used in Cardiovascular Treatment with EPR Spectroscopy}

\begin{abstract}
In this study, the nature of free radicals produced in losartan potassium and indapamide was determined using electron paramagnetic resonance (EPR) spectroscopy at room temperature. The EPR spectra of these samples were attributed to the $\mathrm{CHC} \mathrm{HCH}_{3}$ and $\dot{\mathrm{C}} \mathrm{HCHCH}_{3} \mathrm{NH}$ radicals, respectively. The hyperfine coupling constants and g-values of the free radicals were calculated. EPR measurements show that the stability of the paramagnetic center after radiation and the spin intensity depend on the dose of absorbed radiation. The paramagnetic centers in both samples were determined by considering the chemical structure of the samples. The magnetic properties of the two materials and the possible structure of the paramagnetic center are discussed in this study.
\end{abstract}

Keywords: EPR, Gama-Iradiation, Paramagnetic Centre

\section{Giriș}

Radyasyonun canlılar üzerinde önemli hasarlar oluşturduğu bilinmektedir. Radyasyona tutulan canlılarda oluşan bozukluğun cinsi ve ömür süresi merak ve araştırma konusudur. Biyolojik maddelerde oluşan bozukluklarla inorganik ve organik maddelerde oluşan bozukluklar arasında benzerlik bulunmaktadır. Örneğin kanserli dokuların verdiği EPR spektrumlarının radyasyona tutulmuş maddelerin verdiği EPR spektrumlarına benzemesi bu tür çalışmaların önemini artırmıştır. Radyasyon ile ilaç
(Madde) etkileşmesinde, radyasyonun ilaçlarda oluşturduğu fiziksel ve kimyasal değişimlerin yanında, ayrıca oluşan paramanyetik yapının (Serbest radikallerin) kararlılığı da çok önemlidir (Nakagawa vd., 2008). Elektron paramanyetik rezonans (EPR) spektrokopisi, radyasyon ilaç etkileşmesi sonucu ilaçta oluşan paramanyetik merkezin (Serbest radikallerin) elektronik yapısı ile ilgili faydalı bilgiler elde etmek için çok hassas bir metodtur (Osmanoğlu 2017; Osmanoğlu 2008; Basly 1999; Ambroz vd., 2002). Nükleer ve 
elektronik manyetik momentler arasındaki EMX081 X-band EPR spektroskopisi ile etkileşmeyi karakterize eden aşırı ince yapı sabitleri EPR spektrumlarında aşırı ince yapı yarılması olarak tanımlanır (Ogawa vd., 1980; Osmanoğlu vd., 2005; Bhat ve Sridhar 2011). Radyasyona maruz birakılan canlıların yapı taşı olan amino asitlerde,bileşiklerinde ve ilaçlarda radyasyon bozulma merkezleri (Serbest radikaller) gözlenmiştir (Sütçü vd., 2014; Talbi vd., 2004; Stuglik vd., 2008; Aydın 2010). Damian ve arkadaşları tarafindan, gama radyasyonuna tutulan $\mathrm{B}_{3}$ vitamininde oluşan paramanyetik merkezin, kararlılığı ve davranışı incelenmiş, ayrıca bazı spektroskopik özellikler ve paramanyetik merkezin yapısı EPR spektroskopisi kullanılarak tartışılmıştır (Damian vd., 2005). Mikro kristal toz formundaki thiazide ilacı, gama radyasyonuna tutulmuş ve radyasyonun bu ilaçta oluşturduğu bozulma merkezleri (serbest radikaller) incelenmiş [Latosinska ve Pietrzak 2004]. Losartan potasyum ve indapamid, kalp-damar tedavisinde kullanılan ilaçlardır. Bu ilaçların, yapılan literatür araştırmalarında daha önceden EPR veya ESR çalışmasının yapılmadığı görülmüştür. $\mathrm{Bu}$ nedenle, kalp-damar tedavisinde kullanılan bu ilaçların EPR incelenmesini uygun gördük. Bu örneklerin radyasyona tutulmadan önce herhangi bir paramanyetik (radikal) özellik göstermediği, radyasyona tutulduktan sonra paramanyetik özellik kazandığı EPR spektroskopisiyle belirlenmiştir.

\section{Materyal ve Metot}

Tüm hastalıkların hamisi serbest radikaller olduğu için tıp’ta tedavi amaçlı kullanılan ilaçlarda serbest radikal üzerine çalışmalarımızı yoğunlaştırdık. Kalp-damar tedavisinde kullanilan losartan potasyum ve indapamid ilaçları ticari firmalardan temin edildi. $\mathrm{Bu}$ ilaçların mikro kristal toz örnekleri, Atom Enerjisi Kurumunda 60Co gama kaynağında toplamda $20 \mathrm{kGy}$ olacak şekilde, yine aynı kurumda, Bruker model aşagıdaki koşullar belirlenerek spektrum alma işlemleri gerçekleştirilmiştir.

mikrodalga frekansı: $9.7 \mathrm{GHz}$

mikrodalga gücü: $2 \mathrm{~mW}$

modülasyon frekansı: $100 \mathrm{kHz}$

modülasyon genişliği: $0.3 \mathrm{mT}$

zaman sabiti: $1310.720 \mathrm{~ms}$

$\mathrm{Bu}$ koşullar göz önüne alınarak, oda sıcaklığında alınmıştır. EPR ölçümleri alınan örneklerin kararlılığını belirlemek için 4 ay boyunca belirli aralıklarla ölçümler alınmıştır. Alınan EPR spektrumlarının doğruluğunu test etmek için McKelvey'in mikro kristal toz simülasyon programı kullanılmıştır (McKelvey ve Huckel 1987). Spektroskopik g faktörünün doğru ölçümü için DPPH (2,2-difenil-1-picrylhydrazyl) kullanılarak doğru bir karşılaştırma yapılmıştır. Her iki örneğe ait kimyasal yapılar Tablo 1'de verilmiştir.

Tablo 1. Losartan potasyum ve indapamide örneklerinin kimyasal yapıları.

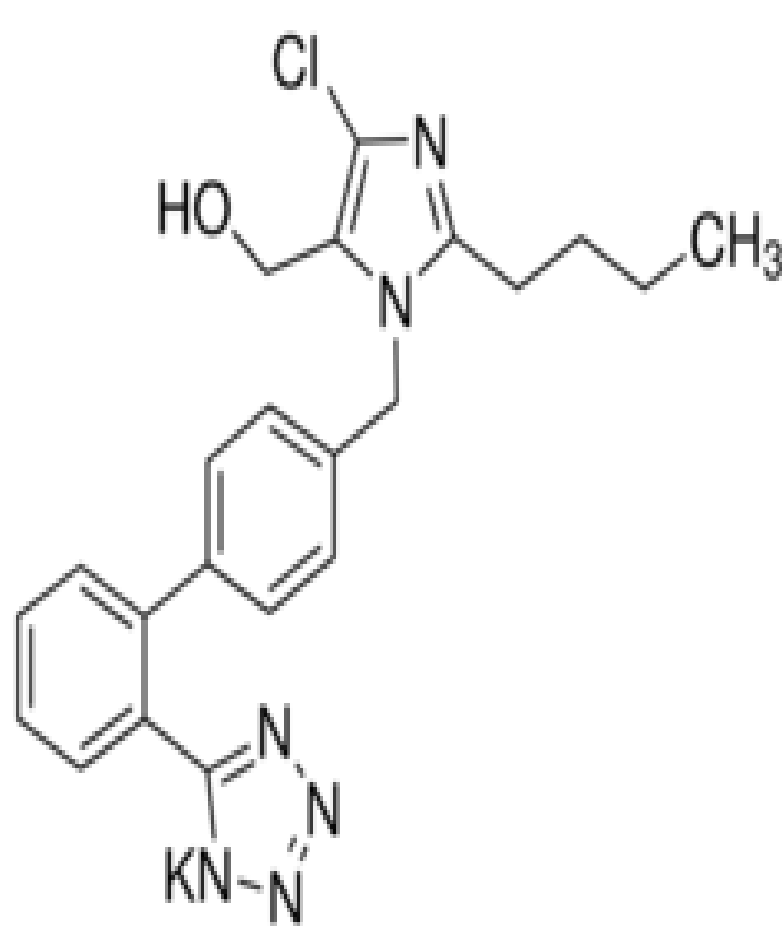

Losartan potasyum'un kimyasal yapısı 


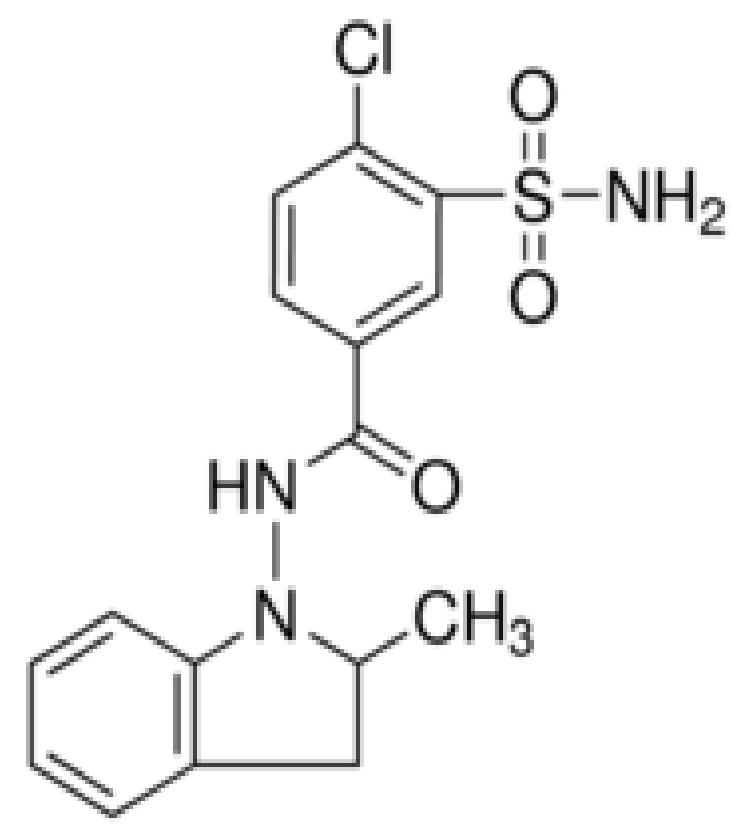

İndapamide'nin Kimyasal yapıs1

\section{Bulgular}

Gama radyasyonuna tutulmuş losartan potasyumun mikro kristal toz formu için oda sıcaklığında alınan deneysel spektrum şekil 1a'da verilmiştir.

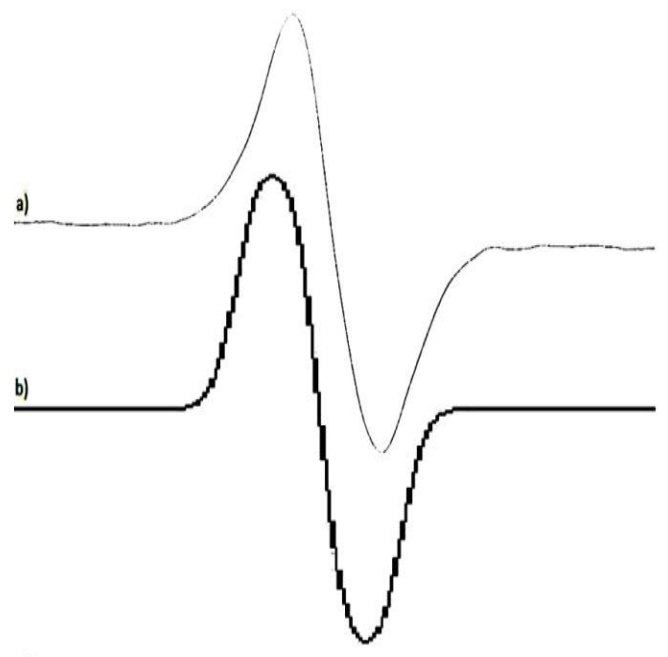

c)

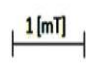

Şekil 1. (a) Işınlanmış losartan potasyumun deneysel EPR spektrumu (b) Losartan potasyumun simülasyon spektrumu (c)Işınlanmamış losartan potasyumun deneysel EPR spektrumu.

Şekilla da verilen spektrum merkezi geniş bir sinyalden ibarettir. Spektrumun genişlemesine dipolar ve spin-spin etkileşmesinin katkısı büyüktür. Simülasyon spektrumu bu katk1lar1 göstermektedir. Radyasyondan önce, EPR sinyallerinin gözlenmediği Şekillc'de verilmiştir. EPR sinyallerinin gözlenmemesi Losartan potasyum'un manyetik dipol momentinin sıfır olduğunu göstermektedir (Diamanyetik madde, $\mu=0$ ). Radyasyona tutulan örnek'te (ilaç'ta) bir sinyalin oluşması bu örneğin paramanyetik hale geldiğini göstermektedir (Şekilla). Oluşan sinyalin çizgi genişliği $(\Delta \mathrm{H}=0.5 \mathrm{mT})$ oldukça büyük bir değer, ayrıca, aşırı ince yapı sabitleri çok büyük değil ve birbirine yakın değerler. Bu nedenle, aşırı ince yapı yarılmaları çözülemediğinden net olarak spektrumda gözlenmemektedir. Losartan potasyum mikro kristal formunda olduğundan, McKelvey toz simülasyon metodu kullanılarak hesaplanan, $\mathrm{a}^{3} \mathrm{CH}=0.7$ $\mathrm{mT}, \mathrm{a}^{4} \mathrm{CH}=0.8 \mathrm{mT}$ ve $\mathrm{a}^{5} \mathrm{CH}=0.4 \mathrm{mT}$, a.i.y. sabitleri ile bulunan simüle spektrum, deneysel olarak elde edilen spektruma benzer olduğu Şekillb'de görülmektedir. $\mathrm{Bu}$ benzerlik, simülasyon metoduyla elde edilen a.i.y. sabitlerinin deneysel spektrumu temsil edebilir anlamına gelmektedir. Deneysel spektrumdan ölçülen spektroskopik g değeri $\mathrm{g}=2.0081$ 'dir. $\mathrm{Bu}$ değer karbon merkezli paramanyetik merkez için karakteristiktir.Bu çalışmada elde edilen a.i.yapı sabitleri R.Köseoğlu ve arkadaşları tarafindan Pentoxifylline (Pentox) ilacı için hesaplanan $\mathrm{a}^{1}=\mathrm{a}^{2}=0.7 \mathrm{mT}, \mathrm{a}^{1}{ }_{\beta}=\mathrm{a}^{2}{ }_{\beta}=0.65 \mathrm{mT}$ a.i.yap 1 sabitlerine yakın değerdedir.(Köseoğlu vd., 2003). Spektroskopik g değeri, paramanyetik merkezin durumunu açıkladığı için oldukça önemlidir. Simüle spektrumundan Belirlenen a.i.y. değerleri ve spektroskopik g değeri, radyasyona tutulan losartan potasyumda oluşan paramanyetik merkezin (radikal'in), $\mathrm{CHC}^{\mathrm{C}} \mathrm{HCH}_{3}$ olduğunu göstermektedir. $\mathrm{Bu}$ paramanyetik merkez, alkil radikalini doğruluyor (Cozar vd., 1997; Osmanoğlu,Ş 2008). İndapamide örneğinin oda sıcaklığındaki deneysel spektrumunda, çizgi genişliği $\Delta \mathrm{H}=0.52 \mathrm{mT}$ olan geniş merkezi tek bir sinyal gözlenmektedir (şekil 2a). Her iki örnekte gözlenen spektrum gaussiyen çizği şeklindedir. Yani, çiflenmemiş elektron 
birden fazla protonla etkileşmektedir. Çünkü, spektrum çizgileri üst üste gelerek spektrum çizgilerine, dipolar etkileşme ve süperpozisyon oluşturduklarından, Aşırı ince spin-spin etkileşmelerinin katkıları yapı yarılmaları çözülmemektedir. gelmektedir. Şöyle'de diyebiliriz; örnekteki etkileşmelerin sayısı, EPR absorpsiyon eğrisi altında kalan alan'la orantılıdır.

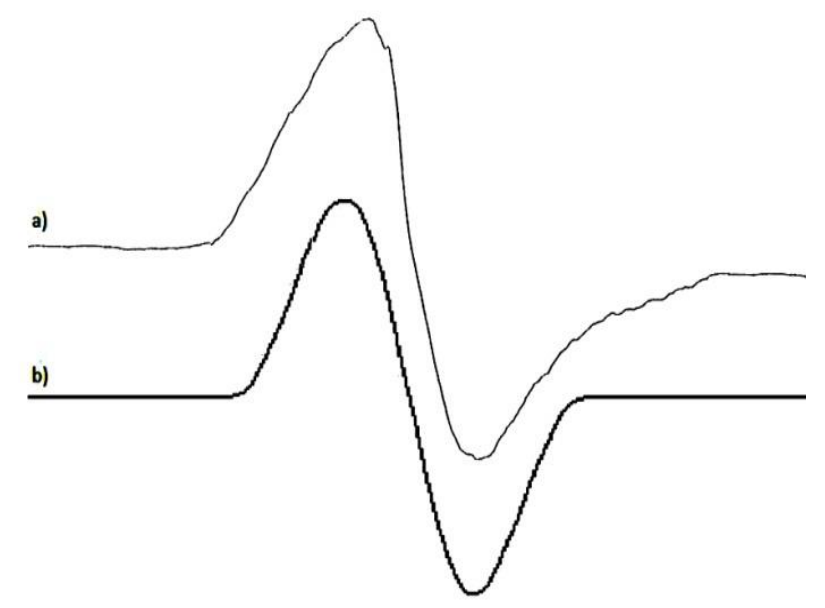

c)

Şekil 2. (a) Işınlanmış indapamide deneysel EPR spektrumu (b) İndapamidine simülasyon spektrumu (c) Radyasyona tutulmamış(Işınlanmamış) indapamide örneğinin deneysel EPR spektrumu Şekil 2c'de sunulmuştur.

Deneysel spektrumda, paramanyetik merkezin durumunu gösteren spektroskopik $\mathrm{g}$ faktörü $\mathrm{g}=2.0059$ olarak ölçülmüştür. Simülasyon metoduyla bulunan a.i.y. sabitleri, sirasiyla, $\mathrm{a}_{\alpha}=1.8 \mathrm{mT}, \mathrm{a}_{\beta}=1.35 \mathrm{mT}$, $\mathrm{a}^{1}{ }_{\gamma}=0.86 \mathrm{mT}, \mathrm{a}^{2}{ }_{\mathrm{\gamma}}=0.75 \mathrm{mT}, \mathrm{a}^{3}{ }_{\mathrm{\gamma}}=0.47 \mathrm{mT}$ ve $a_{N}=0.76 \mathrm{mT}^{\prime}$ dır. Lamotrijin ilacı için yapılan EPR incelenmesinde hesaplanan manyetik parametreler sirasiyala: $\mathrm{a}^{1} \mathrm{CH}=1.7$ $\mathrm{mT}, \mathrm{a}^{2} \mathrm{CH}=1.3 \mathrm{mT}, \mathrm{a}^{3} \mathrm{CH}=0.83 \mathrm{mT}, \mathrm{a}^{2} \mathrm{NH}=$ $0.72 \mathrm{mT}, \mathrm{a}^{1} \mathrm{CNH}=0.43 \mathrm{mT}, \mathrm{a}_{\mathrm{N}}=0.7 \mathrm{mT}$, $\Delta \mathrm{H}=\quad 0.5 \quad \mathrm{mT} \quad$ (Osmanoğlu,Ş 2016) Lamotrijin için EPR çalışmasında elde edilen manyetik parametreler ve spektrum, indapamide için tespit edilen spektruma ve manyetik parametrelere benzerdir. Çiftlenmemiş elektronun komşu protonlarla aşırı ince yapı etkileşmesi sonucu oluşan a.i.y. yarılmaları spektrumda gözlenmemektedir. Bunun nedeni a.i.y. sabitlerinin ve çizgi genişliğinin birbirine yakın değerde olmaları ve dolayısıyla
İndapamide'nin (örneğin) radyasyondan önce alınan spektrumunda hiçbir sinyal gözlenmezken (Şekil 2c), radyasyona maruz bırakıldıktan sonra materyalde, Şekil 2b'de görüldüğü gibi geniş merkezi bir sinyal gözlenmiştir. Spektrum üzerinde yapılan 4 aylık test ölçümleri sonucu indapamide'de oluşan paramanyetik merkezin kararlı olduğu tespit edildi. Radyasyon sonucu, indapamide molekülünde $\mathrm{CH}_{2}$ grubundan $\mathrm{H}$ atomunun ayrılması sonucu yapıda oluşan paramanyetik merkezin (radikal'in), $\dot{\mathrm{C}} \mathrm{HCHCH}_{3} \mathrm{NH}$ olduğunu söyleyebiliriz. $\mathrm{H}$ atomunun ayrılması sonucu, karbon merkezli paramanyetik yapıların oluşması sık görülen bir mekanizmadır (Köksal ve Osmanoğlu,Ş. 1993; Osmanoğlu,Ş. vd., 2002; Başkan ve Osmanoğlu 2004). Hesaplanan a.i.y. sabitleri ve ölçülen $g$ değeri amino alkil radikalini göstermektedir (Aydın) 2010; Köksal vd., 1997; Wood ve Lloyd 1970; Sütçü ve Osmanoğlu,Y. Emre 2017; Osmanoğlu, Y. Emre vd., 2017). Gama radyasyonu sonucu elde edilen deneysel spektrum ile simülasyon metoduyla elde edilen spektrum birbiriyle uyuşmaktadır. Bu uyuşma a.i.y. sabitlerinin doğru hesaplandığını göstermektedir.

\section{Sonuç ve Tartışma}

Radyasyona tutulduktan (1şınlamadan) sonra katı haldeki örneklerde (amino asit,ilaç,gıda) oluşan serbest radikaller oda sıcaklığında araştırıldı. Örneklere ait aşırı ince yapı sabitleri ve çizgi genişlikleri deneysel ve simüle spektrumlar yardımıyla elde edilmiştir. $\mathrm{Bu}$ çalışmada belirlenen spektroskopik parametreler ve radikal yapıları literatürdeki önceden elde edilen sonuçlarla uyumludur. Amino asitlerde elde edilen radikallerin manyetik özelliklerinin araştırılması, biyolojik sistemlerde oluşturulan benzer radikaller için faydalı olabilir. 


\section{Kaynaklar}

Ambroż, H.B., Kornacka, E.M., Marciniec, B., Przybytniak, G. 2002. Radical Decay in Irradiated Drugs: Flutamide, Ifosfamide. Journal of Radioanalytical and Nuclear Chemistry, 254(2), 293-298.

Atherton, N.M. 1973. Electron Spin Resonance Theory and Application, JohnWiley and sons, NewYork.

Aydin, M. 2010. EPR Study of Free Radicals in Amino Acid Derivatives GammaIrradiated at $300 \mathrm{~K}$. Indian Journal of Pure and Applied Physic, 48, 611-614.

Aydin, M. 2010. Identification of Free Radicals Induced by Gamma Irradiation in Amino Acid Derivatives. Brazilian Journal of Physics, 40(3), 306-308.

Basly, J.P. 1999. Radiation Induced Effects on Cephalosporins: An ESR Study. International Journal of Radiation Biology, 75(2), 259-263.

Basly, J.P. 1998. Radiation Induced Effects on Cephalosporins: An ESR Study, International Journal of Radiation Biology, 75(2), 259-263.

Başkan, M.H., Osmanoğlu, Ş. 2004. EPR of Gamma Irradiated Na-Monochloroacetyl$\alpha$-Aminoisobutyric Acid. Zeitschrift für Naturforschung A, 59(10), 665-668.

Bhat, R., Sridhar, K.R. 2011. Influence of Ionizing Radiation and Conventional Food Processing Treatments on the Status of Free Radicals in Lotus Seeds: An ESR Study. Journal of Food Composition and Analysis, 24(4-5), 563-567.

Cozar, O., Chis, V., David, L., Damian, G., Barbur, I. 1997. ESR Investigation of Gamma-Irradiated Aspirin. Journal of Radioanalytical and Nuclear Chemistry, 220(2), 241.

Damian, G., Schmutzer, G., Petrisor, D., Miclauş, V., Simon, S. 2005. Electron Spin Resonance Studies of $\gamma$-Irradiated $\mathrm{B}_{3}$ Vitamin. Romanian Journal of Biophysics, 15(1-4), 23-28.

Köksal, F., Osmanoğlu, Ş., Kartal, I., Ucun, F. 1997. EPR of Gamma Irradiated NaAcetyl L-Glutamic Acid and No-Acetyl
L-Glutamine, Radiation. Physics and Chemistry, 49(5), 537-539.

Köksal, F., Osmanoğlu, Ş. 1993. Electron Paramagnetic Resonance of Gamma Irradiation Damage Centres in Ethylenediaminetetraacetic Acid and Diethylenediaminepentaacetic Acids. Journal of Chemical Research, (2), 84-85.

Köseoğlu, R., Köseoğlu, E., Köksal, F. 2003. Electron paramagnetic resonance of some $\gamma$-irradiated drugs. Applied radiation and Isotopes, 58, 6368.

Latosinska, J.N., Pietrzak, J. 2004. EPR Study Of Free Rdicals in $\mathrm{V}$-İrradiated Thiazide Compounds, Applied Magnetic Resonance, 26(3), 345-355.

McKelvey, R.D., Huckel, M.O. 1987. Theory and Electron Spin Resonance in the Spectroscopy Course. Journal of Chemical Education, 64, 497

Nakagawa, K., Ikota, N., Sato, Y. 2008. Heavy-Ion-Induced Sucrose and L- $\alpha$ Alanine Radicals Investigated by EPR. Applied Magnetic Resonance, 33, 111116.

Ogawa, M., Ishigure, K., Oshima, K. 1980. ESR Study of Irradiated Single Crystals of Amino Acids-1. Radiation Physic and Chemistry, 16, 281-287.

Osmanoğlu, Ş. 2017. Investigation of Radiosensitivity of Gamma Irradiated Procaine Hydrochloride in the Solid State. El-Cezeri Journal of Science and Engineering, 4(3), 314-318.

Osmanoğlu, Ş. 2016. Radiation Effects on Lamotrigine and Flourbiprofen: an EPR stud., El-Cezeri Journal of Science and Engineering, 3, 281-286.

Osmanoğlu, Ş. 2008. Electron Paramagnetic Resonance of Radiation Damage of $\gamma$ irradiated Diethylmalonic Acid. Journal of Molecular Structure, 877(1-3), 7-9.

Osmanoğlu, Ş., Aydın, M., Başkan, M.H. 2005. EPR of Gamma-Irradiated LGlutamine Hydrochloride and NCarbamoyl-L-Glutamic Acid. Zeitschrift für Naturforschung A, 60(7), 549-553.

Osmanoğlu, Ş., Başkan, M.H., Güner, T. 2002. EPR of Gamma Irradiated Single 
Crystal Of 2, 2-Dimethyl Succinic Acid. Zeitschrift für Naturforschung A, 57(11), 909-911.

Osmanoğlu, Y.E., Sütçü, K. 2017. EPR Studies of the Free Radicals Generated in Gamma Irradiated Amino Acid Derivatives. Journal of Molecular Structure, 1145, 240-243.

Osmanoğlu, Y.E., Sütçü, K., Başkan, M.H. 2017. EPR Investigation of GammaIrradiated L-Citrulline, A-Methyl-DlSerine, 3-Fluoro-Dl-Valine and N-AcetylL-Cysteine. Journal of Molecular Structure, 1130, 291-295.

Peimel-Stuglik, Z., Kowalak, S., Jankowska, A. 2008. EPR Spectra of $\gamma$-İradiated D1A-Alanine Supported on Molecular Sieves. Spectrochimica Acta Part A: Molecular and Biomolecular Spectroscopy, 69(5), 1395-1404.

Poole, C.P., Farach, H.A. 1967. The theory of magnetic resonance, first edition elsevier puplishing company, Amsterdam.

Sütçü, K., Osmanoğlu, Y.E. 2017. EPR Study of Gamma-Irradiated N-Methyl-LAlanine, DL-2-Methyl Glutamic Acid Hemihydrate and Di-Leucine Hydrochloride in Solid State. Journal of Molecular Structure, 1149, 593-595.

Sütçü, K., Osmanoğlu, Y. E., Osmanoğlu, Ş. 2014. Effect of Irradiaiton Dose, Microwave Power, and Storage Time on the Free Radical Concentration in $\gamma$ Irradiated Black Olive (Olea europaea) Seeds. International Journal of Scientific Research and Innovative Technology, 1(3), 21-29.

Talbi, S., Raffi, J., Aréna, S., Colombani, J., Piccerelle, P., Prinderre, P., Dolo, J.M. 2004. EPR Study of Gamma Induced Radicals in Amino Acid Powders. Spectrochimica Acta Part A: Molecular and Biomolecular Spectroscopy, 60(6), 1335-1341.

Wood, D.E., Lloyd, R.V. 1970. EPR of Free Radicals in an Adamantane Matrix. I. Aliphatic Aminoalkyl Radicals. The Journal of Chemical Physics, 53(10), 3932-3942. 